\title{
Environment-Friendly Magnetic Fluids for Wastewater Remediation - Synthesis and Characterization
}

\author{
C. NADEJDE ${ }^{a, *}$, M. NEAMtU ${ }^{a}$ AND D. CREANGA ${ }^{b}$ \\ ${ }^{a}$ Interdisciplinary Research Department - Field Science, Alexandru Ioan Cuza University, \\ Lascar Catargi 54, 700107, Iasi, Romania \\ ${ }^{b}$ Faculty of Physics, Alexandru Ioan Cuza University, Carol I Bd. 11A, 700506, Iasi, Romania
}

\begin{abstract}
Magnetic nanostructured materials have been found to be very efficient in wastewater decontamination. Among the various synthesis methods, co-precipitation is a rapid and cost-effective technique for the manufacturing of magnetic nanoparticles; moreover, using green chemistry for their stabilization with natural non-toxic capping agents in aqueous solution, such materials become potential candidates as nanocatalytic formulations for water remediation, topic which represents the aim of the present study. Thus, three types of environment-friendly magnetic fluids were synthesized using the chemical precipitation route, the resulted samples being further characterized by various analytical techniques, in order to assess their microstructural features. The results revealed excellent stability in suspension of all samples, the magnetite nanoparticles exhibiting sizes in the nanoscale range with a relatively spherical shape and suitable magnetic properties. Such nanoparticle based products could be the right choice for magnetic responsive and recyclable materials used in the degradation of emerging pollutants from wastewaters.
\end{abstract}

DOI: 10.12693 /APhysPolA.127.647

PACS: 81.16.Be; 81.07.Bc; 68.43.-h; 75.50.Mm; 89.60.Ec

\section{Introduction}

Nowadays, the abundance and persistance of a wide range of harmful industrial and personal care products released in the environment is dramatically increasing. In particular, water pollution has become a critical issue worldwide and treatment technologies are in continuous improvement. Nanomaterials were already considered as an efficient strategy for water decontamination [1, 2]; in the efforts for environment protection, it is essential that the treatment methodologies themselves should not contribute with additional harmful materials but should use instead non-toxic biodegradable ones. Many innovative nanomaterials have already being designed using green chemistry allowing their safe use in a wide range of applications, including water remediation [3].

Iron oxide based nanostructures have gained extensive attention in this regard $[1,2]$ since they exhibit excellent unique properties, being known for their enhanced magnetic properties and lack of toxicity. Nowadays, a wide variety of magnetic nanoparticle (NP) synthesis methods have been reported, using either the top-down or the bottom-up strategies [4] and leading to nanostructures with tailored properties for specific applications. Although each method has its own advantages, chemical precipitation is still the most preferred route for submicron magnetic particles yielding, such as magnetite $\left(\mathrm{Fe}_{3} \mathrm{O}_{4}\right)$ NPs.

In environmental applications such as water decontamination, the efficiency of iron oxide NPs as nanocatalysts could be enhanced if they were used as aqueous colloidal

*corresponding author; e-mail: claudia.nadejde@uaic.ro suspensions (ferrofluids) and not in the powdered form, since they can be tailored to remain well-dispersed in aqueous solution by surface stabilization with an appropriate surfactant.

In this communication, we describe an environmentfriendly, facile and cost-effective protocol for ferrofluid synthesis by chemical precipitation, using natural occurring molecules as capping and stabilizing agents for the homogenous dispersion of magnetite NPs in aqueous solution, as potential candidates for water remediation.

The purpose of the study was to examine citric acid, tannic acid and a polyphenolic green tea extract ability for the surface modification and long-term stabilization of iron oxide NPs in aqueous colloidal suspension, while maintaining their good magnetic properties.

\section{Experimental}

\subsection{Reagents and materials}

All chemicals were analytical high purity reagents (Sigma-Aldrich) used in experiments without further purification. Citric acid monohydrate and tannic acid were also purchased from Sigma, while the green tea extract was freshly prepared in laboratory prior to its use, by keeping $10 \mathrm{mg}$ of green tea (commercial product, China) in $50 \mathrm{ml}$ hot water $\left(70^{\circ} \mathrm{C}\right)$ for 30 minutes; the resulted concentrated polyphenolic extract was then filtered and ready to use.

Deionized water (18.2 $\mathrm{M} \Omega / \mathrm{cm}$, Barnstead EASYpure@ II Water Purification System) was used in all experimental procedures.

\subsection{Synthesis of magnetic fluids}

First, superparamagnetic $\mathrm{Fe}_{3} \mathrm{O}_{4} \mathrm{NPs}$ were synthesized by a modified co-precipitation method [5]. In this multistep process (performed under normal atmospheric conditions), $300 \mathrm{ml}$ of $10.866 \mathrm{~g} \mathrm{FeCl}_{3} \times 6 \mathrm{H}_{2} \mathrm{O}$ solution (134 
$\mathrm{mM})$ and $300 \mathrm{ml}$ of $5.588 \mathrm{~g} \mathrm{FeSO}_{4} \times 7 \mathrm{H}_{2} \mathrm{O}$ solution $(67$ $\mathrm{mM}$ ) were mixed together thoroughly and heated up to $75^{\circ} \mathrm{C}$; after five minutes, $150 \mathrm{ml}$ of $2 \mathrm{M} \mathrm{NaOH}$ solution was added dropwise into the homogeneous hot mixture at constant temperature $\left(75^{\circ} \mathrm{C}\right)$ with vigorous magnetic stirring. Magnetite NPs were formed by a short nucleation event followed by a slow growth process:

$$
\begin{aligned}
& \mathrm{FeSO}_{4}+2 \mathrm{FeCl}_{3}+8 \mathrm{NaOH} \\
& \quad \rightarrow \mathrm{Fe}_{3} \mathrm{O}_{4}+6 \mathrm{NaCl}+\mathrm{Na}_{2} \mathrm{SO}_{4}+4 \mathrm{H}_{2} \mathrm{O}
\end{aligned}
$$

According to the thermodynamics of the above reaction, complete precipitation of $\mathrm{Fe}_{3} \mathrm{O}_{4}$ occurs in an environment at alkaline $\mathrm{pH}$ for the stoichiometric $2: 1$ ratio of $\mathrm{Fe}^{3+}$ and $\mathrm{Fe}^{2+}$ salts. Following precipitation, the dispersion $(\mathrm{pH} \approx 12$ ) turned black (indicating the formation of magnetite nanoparticles) and was kept for another 30 minutes under constant magnetic stirring and elevated temperature in order to ensure complete magnetite crystals maturation. To remove salts and residual products, the precipitate was magnetically separated and rinsed three times with deionised water until a $\mathrm{pH} \approx 7$ was reached.

The resulted magnetic slurry was divided in three equal samples for further stabilization in aqueous solution via a green route, to finally yield stable magnetic fluids. During this step, $3.5 \mathrm{ml}$ of surfactant solution (40\% citric acid (CA), $10 \%$ tannic acid (TA), or $50 \%$ green tea extract (GTE)) was slowly added to each magnetite hydrosol sample followed by homogenization and subsequent deionized water dropwise addition up to $50 \mathrm{ml}$ total volume with constant mechanical stirring (1800 rpm) for one hour, yielding three ferrofluid samples denoted as: F-CA, F-TA and respectively F-GTE; while for the first two samples, the dispersion and stabilization process took place at high temperature $\left(75^{\circ} \mathrm{C}\right)$, the F-GTE sample was stabilized at room temperature $\left(25^{\circ} \mathrm{C}\right)$.

The resulted homogenous fluids were filtered followed by washing with deionized water and magnetic decantation for three times for the removal of unbound surfactant from the carrier fluid. Finally, each sample of black ferrophase was resuspended in $50 \mathrm{ml}$ deionized water, while carefully adjusting the solution $\mathrm{pH}$ to ensure long-term stability of each sample: F-CA $(\mathrm{pH} \approx 6), \mathrm{F}-\mathrm{TA}(\mathrm{pH} \approx 8)$ and F-GTE $(\mathrm{pH} \approx 5.5)$.

\subsection{Characterization}

X-ray diffraction (XRD) analysis (Shimadzu LabX XRD-6000 diffractometer with $\mathrm{Cu} \mathrm{K}_{\alpha}$ radiation, $\lambda=$ $1.54 \AA$ ) was performed on the dried NP powder to obtain information regarding the samples crystallinity.

Scanning electron microscopy (SEM) of each diluted sample was performed by Hitachi S3400-N device to provide qualitative information of the sample morphology.

Vibrating sample magnetometer (VSM) system, Model MicroMag 2900/3900, was used on the powdered samples (resulted following drying the magnetic fluids in vacuum at $40^{\circ} \mathrm{C}$ ) at $298 \mathrm{~K}$ for the measurement of their magnetic properties.

\section{Results and discussion}

Following the above described preparation procedure, approximately $5 \mathrm{~g}$ of uncoated magnetite nanoparticles was obtained. The ferrophase coating and dispersion in aqueous phase was accomplished by a green postsynthesis process using three types of natural occurring compounds (citric acid, tannic acid or green tea extract), preventing iron oxide NPs agglomeration and sedimentation in suspension. The choice of organic agents for magnetic core capping is crucial for both the long term stability of the NPs in the ferrofluid and their efficiency and non-toxicity in the application they were designed for. Citric acid is a well-known stabilizing agent [6], while antioxidant compounds such as tannic acid or high content polyphenols mixture from green tea extract were only more recently taken into consideration as good stabilizing/capping compounds for various types of NPs [3].

From the volume fraction of ferrophase: $\Phi=\left(\rho_{\mathrm{F}}-\right.$ $\left.\rho_{\mathrm{W}}\right) /\left(\rho_{\mathrm{S}}-\rho_{\mathrm{W}}\right)$, where $\rho_{\mathrm{F}}-$ ferrofluid density, $\rho_{\mathrm{W}}-$ carrier liquid (water) density, $\rho_{\mathrm{S}}-$ solid phase density estimated by gravimetric measurements $\left(\Phi_{\mathrm{F}-\mathrm{CA}}=\right.$ $2.78 \%, \Phi_{\mathrm{F}-\mathrm{TA}}=3.82 \%$ and $\left.\Phi_{\mathrm{F}-\mathrm{GTE}}=3.45 \%\right)$, and from the SEM measurements of average particle diameter $\left(D_{\mathrm{F}-\mathrm{CA}}=55 \mathrm{~nm}, D_{\mathrm{F}-\mathrm{TA}}=59 \mathrm{~nm}, D_{\mathrm{F}-\mathrm{GTE}}=\right.$ $62 \mathrm{~nm}$ ), the following volume concentrations $(n)$ of the magnetic nanoparticles in the analyzed samples resulted: $n_{\mathrm{F}-\mathrm{CA}}=3.19 \times 10^{11} \mathrm{ml}^{-1}, n_{\mathrm{F}-\mathrm{TA}}=3.55 \times 10^{11} \mathrm{ml}^{-1}$ and respectively $n_{\mathrm{F}-\mathrm{GTE}}=2.76 \times 10^{11} \mathrm{ml}^{-1}$. After 30 days of storage in the dark and low temperature, all samples were still stable with no sedimentation.

The XRD analysis, carried out on the uncoated black powder resulted following the co-precipitation synthesis, confirmed the magnetite type structure of the obtained sample, the reflection peak positions and relative peak intensities being in good agreement with those reported in the literature [4].

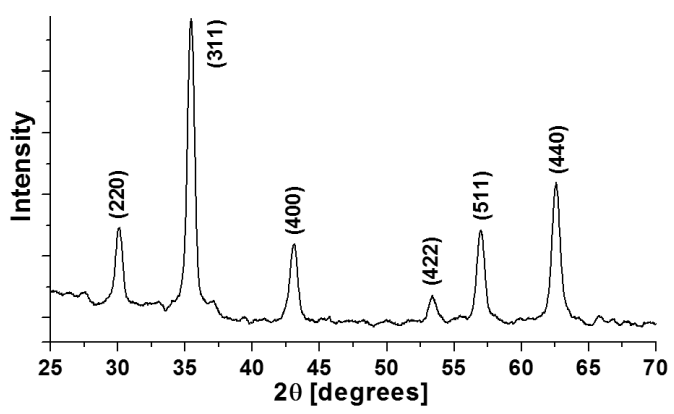

Fig. 1. XRD pattern of uncoated magnetite nanoparticles resulted following co-precipitation route.

The XRD pattern (Fig. 1) indicates the characteristic peaks of highly crystalline $\mathrm{Fe}_{3} \mathrm{O}_{4}$ sample with typical cubic inverse spinel structure and no additional diffraction peaks.

SEM images of each individual sample were also recorded (Fig. 2a-c) in order to check the morphology 

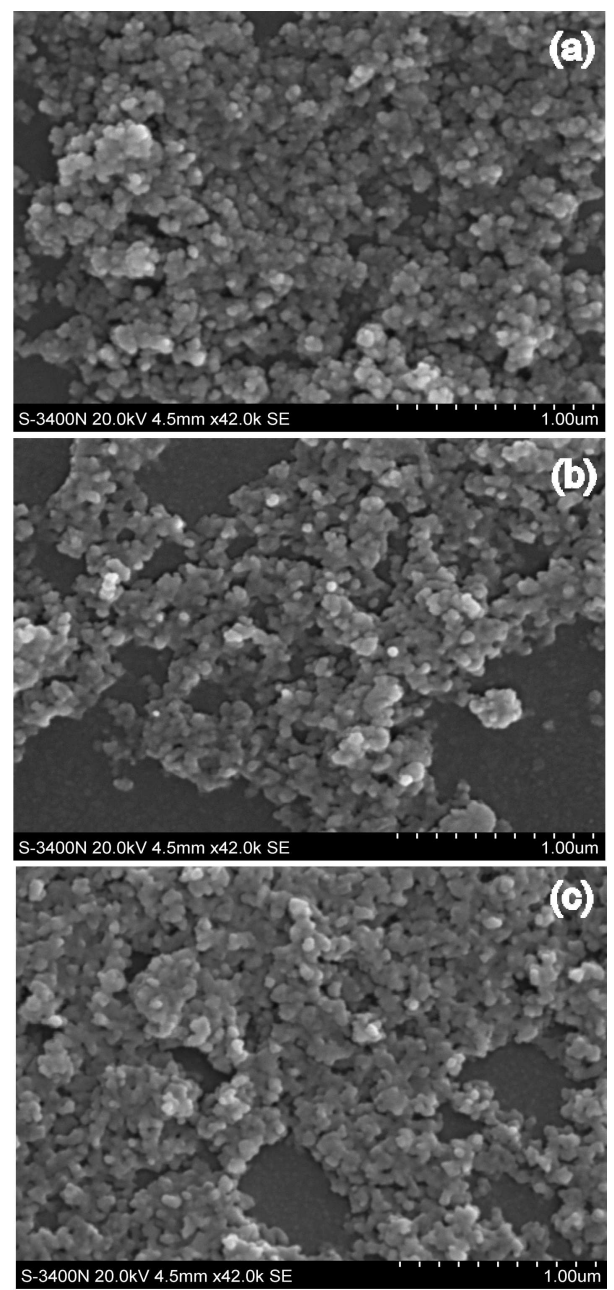

Fig. 2. SEM micrographs of coated magnetite NPs with: a) citric acid; b) tannic acid and c) green tea extract.

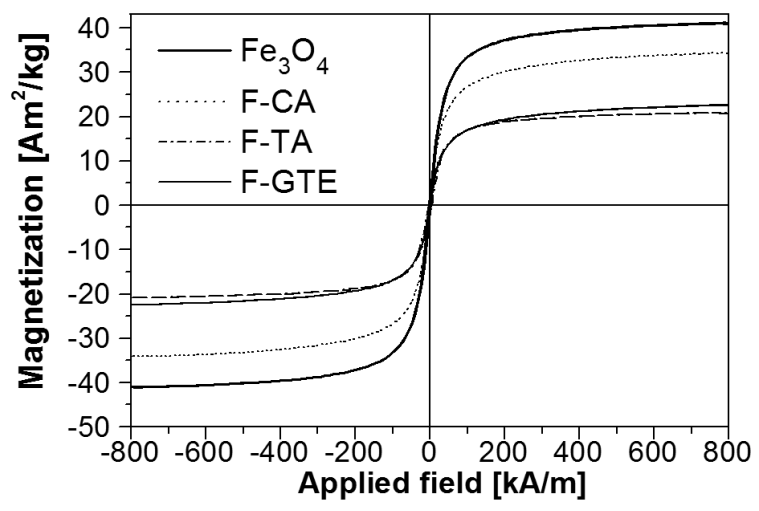

Fig. 3. Magnetization curves of the uncoated $\left(\mathrm{Fe}_{3} \mathrm{O}_{4}\right)$ and coated magnetic NPs (F-CA, F-TA and F-GTE). of the nanoparticles in the ferrofluid samples. All samples are composed of relatively spherical shaped NPs with size below $70 \mathrm{~nm}$, the F-CA sample exhibiting the lowest nanosized dimensions due to the initial acidic $\mathrm{pH}$ of the surfactant added in the stabilization process.

Fig. 3 shows the room-temperature magnetization curves resulted from magnetic measurements of the uncoated and, respectively, capped $\mathrm{Fe}_{3} \mathrm{O}_{4}$ NPs in the samples, which exhibit typical superparamagnetic behaviour with no hysteresis loops. Uncoated $\mathrm{Fe}_{3} \mathrm{O}_{4}$ NPs have the highest saturation magnetization $\left(M_{\mathrm{s}}=\right.$ $40.97 \mathrm{~A} \mathrm{~m}^{2} / \mathrm{kg}$ ), followed by citric acid capped NPs with $M_{\mathrm{s}}=34.25 \mathrm{~A} \mathrm{~m}^{2} / \mathrm{kg}$, while the other two samples present a lower value, of $20.77 \mathrm{~A} \mathrm{~m}^{2} / \mathrm{kg}$ for the tannic acid coated magnetite, respectively of $22.44 \mathrm{~A} \mathrm{~m}^{2} / \mathrm{kg}$ for the green tea extract capped magnetic NPs. It can be concluded that all samples possess good magnetic response, however their magnetism weakens with the increasing shell thickness of the capping agent around the magnetic cores.

\section{Conclusion}

We have successfully synthesized $\mathrm{Fe}_{3} \mathrm{O}_{4}$ NPs based magnetic fluids with good physico-chemical features, their high stability in time being ensured by using a green post-synthesis route for capping the magnetite nanoparticles with non-hazardous natural occurring molecules; moreover, this method is facile, rapid and cost-effective, yielding non-toxic ferrofluids with enhanced magnetic properties, which make them attractive for wastewater treatment applications, as magnetic recoverable nanocatalysts able to convert emerging pollutants into less toxic compounds. The investigations of the synthesized $\mathrm{Fe}_{3} \mathrm{O}_{4}$ NPs in treatability studies are in progress.

\section{Acknowledgments}

This present work was supported by the Romanian Ministry of National Education CNCS-UEFISCDI: project no. PN-II-ID-PCE-2012-4-0477. We also thank Dr. V. Nica for XRD analysis, Dr. S. Tascu for SEM recordings and Dr. P. Postolache for VSM measurements.

\section{References}

[1] P. Xu, G.Mi. Zeng, D.L. Huang, C.L. Feng, S. Hu, M.H. Zhao, C. Lai, Z. Wei, C. Huang, G.X. Xie, Z.F. Liu, Sci. Total Environ. 424, 1 (2012).

[2] S. Rahim Pouran, A.A.A. Raman, W.M.A.W. Daud, J. Cleaner Prod. 64, 24 (2014).

[3] O.V. Kharissova, H.V. Rasika Dias, B.I. Kharisov, B. Olvera Perez, V.M. Jimenez Perez, Trends Biotechnol. 31, 240 (2013).

[4] M. Faraji, Y. Yamini, M. Rezaee, J. Iran. Chem. Soc. 7, 1 (2010).

[5] R.E. Rosenweig, Ferrohydrodynamics, Cambridge University Press, New York 1985.

[6] G. Matei, A. Airinei, D.E. Creanga,Acta Phys. Pol. A. 109, 405 (2006). 